\title{
BMJ Open Environmental management for dengue control: a systematic review protocol
}

To cite: Mahmud MAF, Abdul Mutalip MH, Lodz NA, et al. Environmental management for dengue control: a systematic review protocol. BMJ Open 2019;9:e026101. doi:10.1136/ bmjopen-2018-026101

- Prepublication history and additional material for this paper are available online. To view please visit the journal (http:// dx.doi.org/10.1136/bmjopen2018-026101).

Received 17 August 2018 Revised 15 January 2019 Accepted 8 March 2019

Check for updates

(C) Author(s) (or their employer(s)) 2019. Re-use permitted under CC BY-NC. No commercial re-use. See rights and permissions. Published by BMJ.

${ }^{1}$ Center for Communicable Disease Research, Institute for Public Health, Malaysia, Kuala Lumpur, Wilayah Persekutuan, Malaysia

${ }^{2}$ Environmental Health Institute, (WHO Collaboration Center for Reference and Research of Arbovirus and Their Associated Vectors) National Environment Agency, Singapore, Singapore, Singapore

${ }^{3}$ Institute for Public Health, Kuala Lumpur, Wilayah Persekutuan, Malaysia

Correspondence to Mohd Amierul Fikri Mahmud; amierulfikri@gmail.com

\author{
Mohd Amierul Fikri Mahmud, ${ }^{1}$ Mohd Hatta Abdul Mutalip, ${ }^{1}$ Noor Aliza Lodz, \\ Eida Nurhadzira Muhammad, ${ }^{1}$ Norzawati Yoep, ${ }^{1}$ Mohd Hazrin Hashim, ${ }^{1}$ \\ Faizah Paiwai, ${ }^{1}$ Jayanthi Rajarethinam, ${ }^{2}$ Joel Aik, ${ }^{2}$ Nor Asiah Muhammad ${ }^{3}$
}

\section{ABSTRACT}

Introduction Dengue is among the most important mosquito-borne diseases, with more than half of the world's population at risk of infection in dengue endemic countries. Environmental management, which includes any activities that involve environmental modification, environmental manipulation and changes to human behaviour have been used to mitigate the risk of dengue transmission. In this protocol, we will integrate the data from various sources to assess the overall effect of environmental management on the incidence of dengue and other entomological indices.

Methods and analyses We will conduct a systematic review of intervention that assess the effect of environmental management on the incidence of dengue and/or entomological indices. We will include any studies that include intervention through environmental management for dengue control, involving environmental modification, environmental manipulation and changes to human behaviour. A comprehensive search will be performed in electronic databases PUBMED, CENTRAL, SCOPUS, Web of Science and relevant research websites such as PROPSERO, WHO ICTRP and ClinicalTrials.gov to identify studies that meet our inclusion criteria. A systematic approach to searching, screening, reviewing and data extraction will be applied based on Preferred Reporting Items for Systematic reviews and Meta-Analysis. Titles, abstract, keywords for eligibility will be examined independently by researchers. The quality of the included studies will be assessed using quality assessment tool for studies with diverse design and Cochrane risk of bias tool. The characteristics of the selected articles will be described based on the study design, types of intervention and outcomes of the study in various countries. These include the types of environmental management intervention methods and the effectiveness of the intervention in reducing dengue cases or incidence and impact on entomological indices.

Ethics and dissemination We will register this systematic review with the National Medical Research Register, Ministry of Health Malaysia. This protocol also had been registered with the PROSPERO. No ethical approval is necessary, as there will be no collection of primary data. The results will be disseminated though a peer-reviewed publication and conference presentation. Trial registration number CRD42018092189.

\section{INTRODUCTION}

\section{Background}

Dengue is among the most important mosquito-borne diseases, with more than half

\section{Strengths and limitations of this study}

- We will integrate the data from various sources to assess the overall effect of environmental management on the incidence of dengue and other entomological indices.

- We will summarise the evidences on the effectiveness of environmental management intervention that will provide potential benefits to the service providers, policy makers, researchers and other institutions on dengue control.

- Several studies with statistical drawback where they did not report $p$ values or any statistical description, but merely qualitatively described the impact of their interventions that controlled dengue or Aedes population may limit the quality of the evidences.

of the world's population at risk of infection in dengue endemic countries. The disease is endemic in more than 100 countries with USA, South-East Asia and Western Pacific regions being the most affected. A recent study in $2013^{1}$ indicates that 390 million dengue infections occur every year $(95 \%$ credible interval 284 to 528 million) of which 96 million manifests clinically. Dengue is widespread throughout the tropics, with several risk factors such as rainfall, temperature, relative humidity, degree of urbanisation and quality of vector control services in the areas. Despite the availability of dengue vaccines, few countries can afford to implement its uptake at the national level. Vector control continues to remain an important approach in reducing dengue transmission in many dengue endemic countries. The application of vector control methods, including source reduction, use of chemical larvicides and adulticides and use of biological control is hindered by weak programme capacity, which includes the absence of well-defined indicators and programme targets, poor understanding of efficacy and cost-effectiveness of control measures ${ }^{2}$ in term of reducing dengue transmission. 
Environmental management refers to the change of the environment in order to prevent or minimise vector productivity and human contact with the vector-pathogen by the methods such as destroying, altering, removing or recycling non-essential containers that serve as vector habitats. Based on WHO's definition, ${ }^{3}$ there are three types of environmental management in controlling dengue which consists of (i) environmental modification, (ii) environmental manipulation and (iii) changes to human habitation or behaviour.

Environmental management involves the improvement of water supply and water storage system, ${ }^{45}$ mosquito-proofing of water-storage containers, ${ }^{6}$ solid waste management, community clean-up ${ }^{7}$ and modification of building structures. Environmental management also understands mosquito ecology and population dynamics as well as the epidemiology of mosquito-borne diseases. Notably, environmental management is not intended to replace other vector-borne diseases control methods, but to complement and provide for the development of "integrated control" strategies. ${ }^{8}$

In several countries, environmental management aim to suppress dengue vector population through the provision of safe water supplies, establishing proper sanitation, improve solid waste management facilities, sewerage and excreta disposal systems and water manipulation in dams and irrigation. Others implement cover fitted over concrete rainwater storage tanks to prevent mosquito breeding in key containers and to reduce adult vector densities and longevity. ${ }^{9}$ The element of community participation to apply environmental management intervention methods has been proven effective in reducing infection risk. ${ }^{10}$

Environmental management alone or combined with other methods have proven to be a successful approach to vector control. ${ }^{11}$ A study in Vietnam and Thailand demonstrated the use of community-based environmental management in high reduction of dengue incidence. ${ }^{12} 13$

\section{Why is it important to do this review?}

Since there is no curative treatment for dengue yet, prevention that targets environmental and ecosystem management is increasingly relevant. ${ }^{14}$ To reduce the vector that carries the virus for transmission, three main vector control methods; chemical, biological and environmental control are applied. Environmental management is considered as important component in dengue prevention and control. Many studies have been conducted to investigate the role of environmental management in dengue control which is substantially relevant to reduce dengue incidence in the population. One systematic review synthesised information on one type of environmental control that examined changes to human behaviour, however combination of this method with chemical and biological methods still are not able to reduce dengue incidence. ${ }^{15}$ Other review also examined the four types of dengue control that include biological, chemical, environmental and integrated vector control that could reduce dengue. ${ }^{16}$ Numerous studies have also investigated the effectiveness of individual role of environmental management to control dengue, hence there are contentious findings to control dengue.

However, to our present knowledge, there are no documented reviews that explicitly discuss or syntheses current available evidence on the effectiveness of environmental management interventions for dengue control. In this propose study, we will integrate the data from various sources to assess the overall effect of environmental management on the incidence of dengue and other entomological indices. A comprehensive systematic review could provide the best available evidence on the effectiveness of various types of intervention in environmental management strategy including modification, manipulation and human behaviour. This review is essential to identify the effective environmental management intervention strategy to reduce dengue cases. Information from this review will provide potential benefits to the service providers, policy makers, researchers and other institutions on dengue control.

\section{Objective}

This study aims to systematically evaluate the effect of environmental management in dengue control to reduce dengue incidence and entomological indices.

\section{METHODS}

\section{Criteria for considering studies for this review}

Patient and public involvement

Patients and public were not involved in this study as this is a systematic review protocol.

\section{Types of studies}

We will include all observational studies namely cohort, case-control and cross-sectional. We will also include all randomised control trials (RCT), cluster-RCT, quasi-RCT, control cohort before and after (CChBA) and interrupted time series (ITS) that evaluate the effectiveness of environmental management interventions. We will exclude cross-over studies due to concerns about the 'carry-over' effects. We will also exclude any short communication, guidelines and case report.

\section{Population}

We will include participants of all ages who are involved in environmental management for dengue control programme, whether their involvement is in the community-based activities or individual preventative dengue control programme.

\section{Types of interventions}

We will be looking specifically at these three types of interventions:

1. Environmental modification: Involves long-lasting physical transformations to reduce vector larval habitat.

2. Environmental manipulation: Temporary changes to vector habitats that involves the management of essential containers. 
3. Human behaviour: Changes in human behaviour that involves actions to reduce human-vector contact.

In this review, the comparators are other interventions which do not include a component in single or in combination of environmental management for dengue control such as chemical control, biological control or personal protection.

\section{Types of outcome measures \\ Primary outcomes}

Cases of confirmed dengue as defined by WHO guidelines $^{1718}$ (online supplementary appendix 1 ).

\section{Secondary outcomes}

- Houses positive for larvae and/or pupae; for example, the percentage of houses infested with larvae/pupae with one or more habitats for Aedes aegypti or related species (house index). ${ }^{19}$

- Containers infested with larvae/pupae; for example, the number of positive containers per 100 houses (breteau index). ${ }^{19}$

- Containers specifically designed for water storage infested with larvae/pupae; for example, the percentage of water-holding containers infested with larvae/pupae (container index). ${ }^{19}$

- Number of pupae per 100 houses inspected (pupae index)..$^{19}$

- Any reported adverse outcome or unintended consequences.

\section{SEARCH METHODS FOR IDENTIFICATION}

\section{Electronic searches}

We will systematically conduct a comprehensive literature search using different bibliographic databases. We will conduct electronic searches from selected databases for eligible papers published until 10 January, 2018, without language restrictions. Databases for this review include PubMed, EMBASE, CENTRAL, Web of Science and Scopus. We will also retrieve registered clinical or community trials from PROSPERO, WHO IRTCP and ClinicalTrials.gov to identify unpublished works and ongoing trials related to the scope of this review.

We will adapt the search strategy (online supplementary appendix 2) with the key elements in research question: population, interventions, comparator, outcome and study design (online supplementary appendix 3). Two main approaches for search strategy include searching using medical subject headings terms and free-text terms in the title and abstract on databases. We will include potential studies in English language only for this review. We will also check reference lists from all reviews related to the inclusion criteria and any eligible articles will be checked for duplication.

\section{Selection of studies}

Two review authors will independently examine titles and abstracts of the references and will exclude all the obviously non-relevant studies. We will retrieve the full-text articles for the potentially relevant studies. Two review authors will independently screen the full-text articles and identify studies for inclusion according to the eligibility criteria, and record the reasons for exclusion of the excluded studies. Whenever necessary we will contact the study authors to clarify the eligibility for each study. We will also identify any multiple publications from the same data source and include only data from the main source to avoid duplication. We will resolve any disagreements through discussion or, if necessary, we will consult a third review author. We will record the selection process and complete the Preferred Reporting Items for Systematic Reviews and Meta-Analyses flow diagram. ${ }^{20}$

\section{Data extraction}

Two authors will extract the data and independently complete the data extraction form. We will refer any disagreement to third author. The data extraction form include variables as follows:

- Title, author (year) and location.

- Methods: Study design, duration, setting and analysis.

- Types of environmental management interventions: Modification, manipulation or human behaviour.

- Outcome measures: Primary outcome is impact on dengue incidence, reported dengue fever, dengue haemorrhagic fever or dengue shock syndrome cases. Secondary outcome is based on impact on entomological indices; Aedes index, house index, breteau index, container index and pupae index.

\section{Quality assessment}

All included papers will be evaluated for study quality. For mixed methods studies, we will use the quality assessment tool for studies with diverse design (QATSDD) ${ }^{21}$ There are 14 QATSDD items related to quantitative studies. Each item was rated on a four-point Likert scale from not at all (0), very slightly (1), moderate (2) and complete (3). The maximum score is 42 . The scoring threshold are those over $75 \%$ 'high' quality, those between $50 \%$ and $75 \%$ 'good' and below 25\% 'poor'. For RCT, quasi RCT and cluster RCT studies, we will evaluate the quality of the paper using Cochrane risk of bias tool. ${ }^{22}$

\section{Data synthesis and statistical analysis}

We will pool the data from each study judged to be clinically homogeneous using Review Manager software (RevMan 2014) if we can find sufficient studies available. We will perform a meta-analysis, if more than one study that provide usable data in any single comparison is available. We will stratify the analyses in forest plots and tables by type of studies such as RCTs, cluster RCTs, quasi RCTs, CChBA, ITS and other observational studies and type of interventions. We will present results from other observational studies in additional tables. We will chart and tabulate descriptive summary of the results on environmental management interventions including environmental modification, manipulation and human behaviour. We will describe skewed data and report as medians and IQRs. If we find multiple trial arms in a 
single study, we will include only the relevant arms. We will assemble a collection of the best evidence for environmental management to control dengue and reduce mosquito population via entomological indices. Data will be qualitatively synthesised based on scopes or activities that will include:

- Method or combination of several environmental management methods.

- Explore the effectiveness of the best types for environmental management intervention such as environmental modification, environmental manipulation and changes to human behaviour.

- The best cost-effectiveness of environmental management to control dengue.

\section{Summary of findings table}

We will create a 'Summary of findings' table using the following outcomes: cases of confirmed dengue, house index, breteau index, container index and any reported adverse outcome or unintended consequences. We will justify all decisions to downgrade or upgrade the quality of studies using footnotes, and we will make comments to aid readers' understanding of the review, where necessary.

\section{Reaching conclusions}

We will base our conclusions only on findings from the quantitative and narrative synthesis of included studies for this review. The conclusions can be used as guidelines for health authority in their control and preventative activities. Our implications for research will suggest priorities for future research and outline the remaining uncertainties in the area.

\section{Ethics and dissemination}

We plan to use the findings of this review to provide evidences of the effectiveness of environmental management intervention strategy that will offer potential benefits to the service providers, policy makers, researchers and other institutions on dengue control.

All findings will be disseminated at any local or international conference, including local authorities and Disease Control Division of Ministry of Health Malaysia.

Acknowledgements We would like to thank the Director General of Health Malaysia for allowing us to publish this systematic review protocol. We would also like to thank the National Environment Agency, Singapore (WHO Collaborating Center), for their support and technical guidance in developing this protocol.

Contributors Conceiving the protocol: MAFM and MHAM. Designing the protocol: MAFM, NAL, JA and JR. Coordinating the protocol: MAFM. Designing search strategies: ENM, FP, NY and MHH. Writing the protocol: MAFM, MAHM and NAM. Providing advice on the protocol: NAM.

Funding The authors have not declared a specific grant for this research from any funding agency in the public, commercial or not-for-profit sectors.

Competing interests None declared.

Patient consent for publication Not required.
Provenance and peer review Not commissioned; externally peer reviewed.

Open access This is an open access article distributed in accordance with the Creative Commons Attribution Non Commercial (CC BY-NC 4.0) license, which permits others to distribute, remix, adapt, build upon this work non-commercially, and license their derivative works on different terms, provided the original work is properly cited, appropriate credit is given, any changes made indicated, and the use is non-commercial. See: http://creativecommons.org/licenses/by-nc/4.0/.

\section{REFERENCES}

1. Bhatt S, Gething PW, Brady OJ, et al. The global distribution and burden of dengue. Nature 2013;496:504.

2. Horstick O, Runge-Ranzinger S, Nathan MB, et al. Dengue vectorcontrol services: how do they work? A systematic literature review and country case studies. Trans $R$ Soc Trop Med Hyg 2010;104:379-86.

3. (WHO) WHOEnvironmental management 2018. http://www.who.int/ denguecontrol /control strategies /environmental /en /.

4. Toledo ME, Vanlerberghe $\mathrm{V}$, Baly $\mathrm{A}$, et al. Towards active community participation in dengue vector control: results from action research in Santiago de Cuba, Cuba. Trans $R$ Soc Trop Med Hyg 2007;101:56-63.

5. Vanlerberghe V, Toledo ME, Rodríguez M, et al. Community involvement in dengue vector control: cluster randomised trial. BMJ 2009;338:b1959.

6. Overgaard HJ, Alexander N, Matiz MI, et al. A Cluster-Randomized Controlled Trial to Reduce Diarrheal Disease and Dengue Entomological Risk Factors in Rural Primary Schools in Colombia. PLoS Negl Trop Dis 2016;10:e0005106.

7. Caprara A, Lima JW, Peixoto AC, et al. Entomological impact and social participation in dengue control: a cluster randomized trial in Fortaleza, Brazil. Trans R Soc Trop Med Hyg 2015;109:99-105.

8. Organization WH. Manual on environmental management for mosquito control with special emphasis on malaria vectors. 1982

9. N M. Interview with Key Informant on Dengue Disease Transmission and Environmental Management. Geneva, 2004.

10. Bowman LR, Donegan S, McCall PJ. Is Dengue Vector Control Deficient in Effectiveness or Evidence?: Systematic Review and Meta-analysis. PLoS Negl Trop Dis 2016;10:e0004551.

11. Kay B, Vu SN, Nam VS. New strategy against Aedes aegypti in Vietnam. Lancet 2005;365:613-7.

12. Ault SK. Environmental management: a re-emerging vector control strategy. Am J Trop Med Hyg 1994;50(6 Suppl):35-49.

13. Kittayapong $P$, Thongyuan $S$, Olanratmanee $P$, et al. Application of eco-friendly tools and eco-bio-social strategies to control dengue vectors in urban and peri-urban settings in Thailand. Pathog Glob Health 2012;106:446-54.

14. Organization WH. Better environmental management for control of dengue [Website]. 2018. [updated 1 January 2018; cited 1 January 2018] http://www.who.int/heli/risks/vectors/denguecontrol/en/.

15. Heintze C, Velasco Garrido M, Kroeger A. What do communitybased dengue control programmes achieve? A systematic review of published evaluations. Trans R Soc Trop Med Hyg 2007;101:317-25.

16. Erlanger TE, Keiser J, Utzinger J. Effect of dengue vector control interventions on entomological parameters in developing countries: a systematic review and meta-analysis. Med Vet Entomol 2008;22:203-21.

17. Barniol J, Gaczkowski R, Barbato EV, et al. Usefulness and applicability of the revised dengue case classification by disease: multi-centre study in 18 countries. BMC Infect Dis 2011;11:106.

18. World Health Organization. Dengue: Guidelines for Diagnosis, Treatment, Prevention and Control. Geneva, Switzerland: WHO, 2009.

19. Who D. Guidelines for Diagnosis, Treatment. Prevention and Control. New. Geneva: TDR/WHO, 2009.

20. Moher D, Altman D. PRISMA (Preferred Reporting items for systematic reviews and Meta-Analyses). The Cochrane Collaboration Methods Groups Newsletter 1996;6.

21. Fenton L, Lauckner H, Gilbert R. The QATSDD critical appraisal tool: comments and critiques. J Eval Clin Pract 2015;21:1125-8.

22. Higgins JPT, Green S, eds. Cochrane Handbook for Systematic Reviews of Interventions Version 5.1.0 [updated March 2011]. 2011. The Cochrane Collaboration. http://handbook.cochrane.org. 